\title{
En pacientes no diabéticos de alto riesgo cardiovascular el tratamiento inten- sivo de la presión arterial reduce los eventos cardiovasculares y la mortalidad
}

In non-diabetic patients with high cardiovascular risk intensive treatment of blood pressure reduces cardiovascular events and mortality

\section{Objetivos}

Comparar el beneficio del tratamiento de la hipertensión arterial (HTA) con un objetivo de tensión arterial sistólica (TAS) menor a $120 \mathrm{mmHg}$ versus un objetivo de TAS menor a 140 $\mathrm{mmHg}$ en pacientes con alto riesgo cardiovascular.

\section{Diseño y lugar}

Ensayo clínico controlado aleatorizado y abierto, realizado en 102 centros clínicos (organizados en 5 redes) en Estados Unidos, incluyendo Puerto Rico.

\section{Pacientes}

Se incluyeron, entre 2010 y 2013, individuos con edad igual o mayor a 50 años, TAS entre 130 y $180 \mathrm{mmHg}$ y alto riesgo cardiovascular; este último definido como al menos uno de los siguientes: enfermedad cardiovascular clínica o subclínica (excepto accidente cerebrovascular - ACV), insuficiencia renal crónica (excepto enfermedad poliquística), riesgo cardiovascular a diez años igual o mayor al 15\%; edad igual o mayor a 75 años.

Se excluyeron individuos con diabetes mellitus (DM), ACV previo o enfermedad poliquística renal

\section{Asignación de pacientes e intervención}

Los participantes fueron asignados aleatoriamente a uno de dos grupos: objetivo de TAS menor a $120 \mathrm{mmHg}$ (tratamiento intensivo) u objetivo de TAS menor a $140 \mathrm{mmHg}$ (tratamiento
The SPRINT Research Group, y col. NEJM. 2015;373(22):2103-16. estándar). La aleatorización fue estratificada por sitio clínico. Los participantes y los integrantes del equipo de investigación conocían la rama asignada; no así los evaluadores de los resultados principales.

\section{Medición de resultados principales}

El resultado primario estuvo compuesto por la ocurrencia de infarto agudo de miocardio (IAM), síndrome coronario agudo (SCA) sin infarto, ACV, insuficiencia cardiaca (IC) aguda descompensada o muerte de causa cardiovascular. Los resultados secundarios incluyeron a los componentes individuales del resultado primario, muerte por cualquier causa, resultados renales. El análisis se realizó por intención de tratar.

\section{Resultados principales}

Fueron incluidos en el análisis 9.361 participantes (4.678 en el grupo intensivo y 4.683 en el grupo estándar). Durante el seguimiento, la media de TAS fue $121,5 \mathrm{mmHg}$ en el grupo intensivo y $134,6 \mathrm{mmHg}$ en el grupo de tratamiento estándar. Los grupos requirieron en promedio 2,8 medicaciones vs. 1,8 medicaciones respectivamente. No hubo diferencias estadísticamente significativas en las características demográficas y comorbilidades entre los grupos, exceptuando en el uso de estatinas $(42,6 \%$ en el grupo intensivo vs $44,7 \%$ en el grupo estándar, $\mathrm{P}=0,04)$. La tabla 1 presenta los resultados principales por grupo.

Tabla 1: Resultados primarios y secundarios con diferencias estadísticamente significativas, por grupo de tratamiento.

\begin{tabular}{l|c|c|c}
\multicolumn{1}{c|}{ Tipo de resultado } & $\begin{array}{c}\text { Grupo intensivo } \\
\text { N (\% por año) }\end{array}$ & $\begin{array}{c}\text { Grupo estándar } \\
\text { N (\% por año) }\end{array}$ & $\begin{array}{c}\text { Hazard Ratio } \\
\text { (IC 95\%) }\end{array}$ \\
\hline Resultado primario & $243(1,65)$ & $319(2,19)$ & $0,75(0,64 \mathrm{a} 0,89)$ \\
\hline Muerte cardiovascular & $37(0,25)$ & $65(0,43)$ & $0,57(0,38 \mathrm{a} 0,85)$ \\
\hline Muerte por cualquier causa & $155(1,03)$ & $210(1,40)$ & $0,73(0,60 \mathrm{a} 0,90)$ \\
\hline Insuficiencia cardiaca & $62(0,41)$ & $100(0,67)$ & $0,62(0,45 \mathrm{a} 0,84)$ \\
\hline
\end{tabular}

No hubo diferencias estadísticamente significativas en la tasa de IAM, SCA y ACV entre los grupos. El número necesario a tratar para evitar un resultado primario fue 61 ; para evitar una muerte cardiovascular, 172; y una muerte por cualquier causa, 90.
La intervención del estudio fue detenidamente tempranamente luego de una mediana de seguimiento de 3,26 años debido a las diferencias encontradas en el resultado primario.

Un análisis por subgrupos evidenció diferencias en el resultado primario en los siguientes grupos (tabla 2):

Tabla 2: Análisis por subgrupos con diferencias estadísticamente significativas en el resultado primario, por grupo de tratamiento

\begin{tabular}{l|c|c|c}
\multicolumn{1}{c|}{ Subgrupo } & Grupo intensivo (\%) & Grupo estándar (\%) & Hazard Ratio (IC 95\%) \\
\hline Participantes sin enfermedad cardiovascular previa & 4,0 & 5,6 & $0,71(0,57 \mathrm{a} 0,88)$ \\
\hline Participantes sin IRC previa & 4,0 & 5,7 & $0,70(0,56 \mathrm{a} 0,87)$ \\
\hline Edad mayor o igual a 75 años & 7,7 & 10,9 & $0,67(0,51 \mathrm{a} 0,86)$ \\
\hline Hombres & 5,5 & 7,6 & $0,72(0,59 \mathrm{a} 0,88)$ \\
\hline Raza no negra & 5,6 & 7,3 & $0,74(0,61 \mathrm{a} 0,90)$ \\
\hline
\end{tabular}

Respecto a los efectos adversos, 220 pacientes $(4,7 \%)$ en el grupo de tratamiento intensivo y $118(2,5 \%)$ en el grupo estándar sufrieron eventos adversos graves que fueron clasificados como relacionados con la intervención (HR: 1,$88 ; p<0.001)$. No hubo diferencias estadísticamente significativas en la incidencia de caídas entre los grupos.

La tabla 3 presenta los resultados de los efectos adversos estadísticamente significativos 
Tabla 3: Eventos adversos graves y/o visitas a guardia con diferencias estadísticamente significativas, por grupo de tratamiento.

\begin{tabular}{l|c|c|c|c}
\multicolumn{1}{c|}{ Efecto adverso grave o visita a guardia } & $\begin{array}{c}\text { Grupo intensivo } \\
\text { N (\%) }\end{array}$ & $\begin{array}{c}\text { Grupo Estándar } \\
\text { N (\%) }\end{array}$ & Hazard Ratio & p valor \\
\hline Hipotensión & $158(3,4)$ & $93(2,0)$ & 1,7 & $<0,001$ \\
\hline Sincope & $163(3,5)$ & $113(2,4)$ & 1,44 & 0,003 \\
\hline Anormalidades electrolíticas & $177(3,8)$ & $129(2,8)$ & 1,38 & 0,006 \\
\hline Insuficiencia renal aguda & $204(4,4)$ & $120(2,6)$ & 1,71 & $<0,001$ \\
\hline
\end{tabular}

\section{Conclusiones}

En pacientes no diabéticos con alto riesgo cardiovascular, el tratamiento intensivo de la TA con un objetivo de TAS menor a $120 \mathrm{mmHg}$ (en comparación con un objetivo de TAS menor a $140 \mathrm{mmHg}$ ) resulta en una disminución de eventos cardiovas- culares fatales y no fatales y de muerte por cualquier causa, a costa de una mayor tasa de eventos adversos serios en el grupo de tratamiento intensivo.

Fuente de financiamiento: Institutos Nacionales de Salud de EE.UU. (NIH), Departamento de Veteranos de EE.UU.

Resumido por: María Victoria Salgado. [ Servicio de Medicina Familiar del Hospital Italiano de Buenos Aires. maria.salgado@ hospitalitaliano.org.ar ]

Salgado MV. En pacientes no diabetico de alto riesgo cardiovascular el tratamiento intensivo de la presión arterial reduce los eventos cardiovasculares y la mortalidad. Evid Act Pract Ambul. 2015;18(4):110-111. Oct-Dic. Resumido de: The SPRINT Research Group, y col. A Randomized Trial of Intensive versus Standard Blood-Pressure Control. NEJM. 2015;373(22):2103-16. PMID: 26551272.

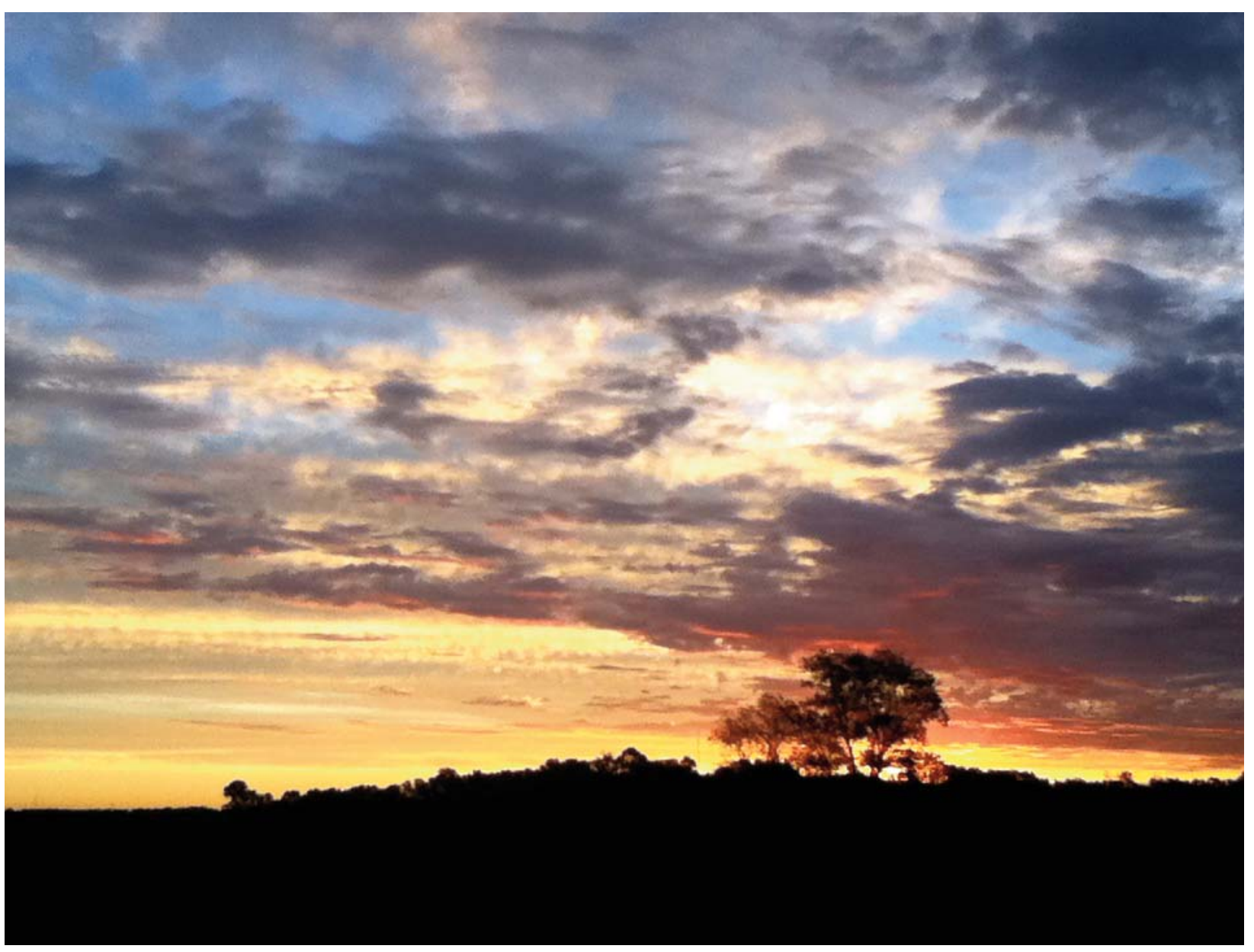

Fotografía: Silvia Spina. 